\title{
Duality results for co-compact Gabor systems
}

\author{
Jakobsen, Mads Sielemann; Lemvig, Jakob
}

Published in:

Proceedings of the 11th International Conference on Sampling Theory and Applications (SampTA 2015)

Link to article, DOI:

10.1109/SAMPTA.2015.7148868

Publication date:

2015

Document Version

Peer reviewed version

Link back to DTU Orbit

Citation $(A P A)$ :

Jakobsen, M. S., \& Lemvig, J. (2015). Duality results for co-compact Gabor systems. In Proceedings of the 11th International Conference on Sampling Theory and Applications (SampTA 2015) (pp. 144-147). IEEE Press. https://doi.org/10.1109/SAMPTA.2015.7148868

\section{General rights}

Copyright and moral rights for the publications made accessible in the public portal are retained by the authors and/or other copyright owners and it is a condition of accessing publications that users recognise and abide by the legal requirements associated with these rights.

- Users may download and print one copy of any publication from the public portal for the purpose of private study or research.

- You may not further distribute the material or use it for any profit-making activity or commercial gain

- You may freely distribute the URL identifying the publication in the public portal

If you believe that this document breaches copyright please contact us providing details, and we will remove access to the work immediately and investigate your claim. 


\section{Duality results for co-compact Gabor systems}

\author{
Mads Sielemann Jakobsen \\ Dep. of Applied Mathematics and Computer Science \\ Technical University of Denmark \\ 2800 Kongens Lyngby, Denmark \\ Email: msja@dtu.dk
}

\author{
Jakob Lemvig \\ Dep. of Applied Mathematics and Computer Science \\ Technical University of Denmark \\ 2800 Kongens Lyngby, Denmark \\ Email: jakle@dtu.dk
}

\begin{abstract}
In this paper we give an account of recent developments in the duality theory of Gabor frames. We prove the Wexler-Raz biorthogonality relations and the duality principle for co-compact Gabor systems on second countable, locally compact abelian groups $G$. Our presentation does not rely on the existence of uniform lattices in $G$.
\end{abstract}

\section{INTRODUCTION}

In Gabor analysis the theory on duality results such as the Wexler-Raz biorthogonal relations and the duality principle plays an important role. The duality results go back to a series of papers in the 1990s [1]-[9] on discrete Gabor systems in $L^{2}(\mathbb{R})$ and $L^{2}\left(\mathbb{R}^{d}\right)$ with modulations and translations along lattices. Recently, these duality results were extended by the two authors [10] to the setting of Gabor systems in $L^{2}(G)$ with modulations and translations along closed, co-compact subgroups, where $G$ is a second countable locally compact abelian (LCA) group. The aim of this note is to give an overview of these extended duality results. We mention that co-compact Gabor systems exist for any LCA group. On the other hand, there exist LCA groups that have no lattices, hence in these cases, the usual duality theory does not apply.

We will give a detailed account of co-compact Gabor systems in Section III and present the extended duality results in Section IV. In Section II we recall some key concepts from frame theory. Let us conclude this introduction with a simple description of co-compact Gabor systems in $L^{2}\left(\mathbb{R}^{d}\right)$.

Example 1. In $L^{2}\left(\mathbb{R}^{d}\right)$ co-compact Gabor systems are of the form

$$
\left\{\mathrm{e}^{2 \pi i \gamma \cdot x} g(x-\lambda)\right\}_{\lambda \in A\left(\mathbb{R}^{s} \times \mathbb{Z}^{d-s}\right), \gamma \in B\left(\mathbb{R}^{r} \times \mathbb{Z}^{d-r}\right)}
$$

for some choice of $0 \leq r, s \leq d, g \in L^{2}\left(\mathbb{R}^{d}\right)$ and $A, B \in G L_{d}(\mathbb{R})$. Depending on the parameters $r$ and $s$, these Gabor systems range from discrete over semi-continuous to continuous families. If we take $r=s=0$, we recover the usual discrete lattice Gabor systems, while the choice $r=s=d$ yields the Gabor system associated with the shorttime Fourier transform.

\section{FRAMES}

A family of vectors $\left\{\varphi_{k}\right\}_{k \in M}$ in a Hilbert space $\mathcal{H}$ is a continuous frame with respect to a measure $\mu$ on the index set $M$ if $k \mapsto \varphi_{k}, M \rightarrow \mathcal{H}$ is measurable and

$$
A\|f\|^{2} \leq \int_{M}\left|\left\langle f, \varphi_{k}\right\rangle\right|^{2} d \mu(k) \leq B\|f\|^{2} \quad \forall f \in \mathcal{H} .
$$

The constants $A$ and $B$ are called frame bounds. If $\left\{\varphi_{k}\right\}_{k \in M}$ is measurable and the upper bound in the above inequality (1) holds, then $\left\{\varphi_{k}\right\}_{k \in M}$ is said to be a Bessel family with constant $B$. A measurable family $\left\{\varphi_{k}\right\}_{k \in M}$ is said to be a tight frame if we can choose $A=B$ in (1). If, furthermore, $A=B=1$, then $\left\{\varphi_{k}\right\}_{k \in M}$ is said to be a Parseval frame.

If $\mu_{M}$ is the counting measure and $\Sigma_{M}=2^{M}$ the discrete $\sigma$-algebra, we say that $\left\{\varphi_{k}\right\}_{k \in M}$ is a discrete frame whenever (1) is satisfied; for this measure space, any family of vectors is obviously measurable.

In the sequel we will use the term frame to mean continuous frame. If $\left\{\varphi_{k}\right\}_{k \in M}$ is a frame, then there exists a dual frame $\left\{\psi_{k}\right\}_{k \in M}$ which is also a frame with respect to the measure space $M$ such that the reproducing formula

$$
f_{1}=\int_{M}\left\langle f_{1}, \varphi_{k}\right\rangle \psi_{k} d \mu(k) \quad \forall f_{1} \in \mathcal{H}
$$

holds weakly, that is,

$$
\left\langle f_{1}, f_{2}\right\rangle=\int_{M}\left\langle f_{1}, \varphi_{k}\right\rangle\left\langle\psi_{k}, f_{2}\right\rangle d \mu(k) \quad \forall f_{1}, f_{2} \in \mathcal{H}
$$

holds. For discrete frames (2) holds in the strong sense with convergence in norm.

A sequence $\left\{\varphi_{k}\right\}_{k \in \mathbb{N}}$ is said to be a Riesz sequence with bounds $A$ and $B$ if

$$
A\left\|\left\{c_{k}\right\}\right\|_{\ell^{2}(\mathbb{N})}^{2} \leq\left\|\sum_{k=1}^{\infty} c_{k} \varphi_{k}\right\|^{2} \leq B\left\|\left\{c_{k}\right\}\right\|_{\ell^{2}(\mathbb{N})}^{2}
$$

for all finite sequences $\left\{c_{k}\right\}_{k \in \mathbb{N}}$.

\section{CO-COMPACT GABOR SYSTEMS}

We let $G$ denote a second countable locally compact abelian group. To $G$ we associate its dual group $\widehat{G}$ which consists of all characters, i.e., all continuous homomorphisms from $G$ into the torus $\mathbb{T} \cong\{z \in \mathbb{C}:|z|=1\}$. Under pointwise multiplication $\widehat{G}$ is also a locally compact abelian group. Throughout the paper we use addition and multiplication as group operation in $G$ and $\widehat{G}$, respectively. By the Pontryagin duality theorem, the dual group of $\widehat{G}$ is isomorphic to $G$ as a topological group, i.e., $\widehat{\widehat{G}} \cong G$. Moreover, if $G$ is discrete, then $\widehat{G}$ is compact, and if $G$ is compact, then $\widehat{G}$ is discrete.

We denote the Haar measure on $G$ by $\mu_{G}$. The Haar measure on any LCA group is unique up to a positive constant. From $\mu_{G}$ we define $L^{1}(G)$ and the Hilbert space $L^{2}(G)$ over the 
complex field in the usual way. The space $L^{2}(G)$ is separable because $G$ is assumed to be second countable. For functions $f \in L^{1}(G)$ we define the Fourier transform

$$
\mathcal{F} f(\omega)=\hat{f}(\omega)=\int_{G} f(x) \overline{\omega(x)} d \mu_{G}(x), \quad \omega \in \widehat{G} .
$$

If $f \in L^{1}(G), \hat{f} \in L^{1}(\widehat{G})$, and the measure on $G$ and $\widehat{G}$ are normalized so that the Plancherel theorem holds (see [11, (31.1)]), the function $f$ can be recovered from $\hat{f}$ by the inverse Fourier transform

$$
f(x)=\mathcal{F}^{-1} \hat{f}(x)=\int_{\widehat{G}} \hat{f}(\omega) \omega(x) d \mu_{\widehat{G}}(\omega), \quad x \in G .
$$

We assume that the measure on a group $\mu_{G}$ and its dual group $\mu_{\widehat{G}}$ are normalized this way, and we refer to them as dual measures.

For $a \in G$, the operator $T_{a}$, called translation by $a$, is defined by

$$
T_{a}: L^{2}(G) \rightarrow L^{2}(G),\left(T_{a} f\right)(x)=f(x-a), \quad x \in G .
$$

For $\chi \in \widehat{G}$, the operator $E_{\chi}$, called modulation by $\chi$, is defined by

$$
E_{\chi}: L^{2}(G) \rightarrow L^{2}(G),\left(E_{\chi} f\right)(x)=\chi(x) f(x), \quad x \in G .
$$

For $t \in L^{\infty}(G)$ the operator $M_{t}$, called multiplication by $t$, is defined by

$$
M_{t}: L^{2}(G) \rightarrow L^{2}(G),\left(M_{t} f\right)(x)=t(x) f(x), \quad x \in G .
$$

The following commutator relations will be used repeatedly:

$$
T_{a} E_{\chi}=\overline{\chi(a)} E_{\chi} T_{a}, \mathcal{F} T_{a}=E_{a^{-1}} \mathcal{F} \text {, and } \mathcal{F} E_{\chi}=T_{\chi} \mathcal{F} \text {. }
$$

For a subset $H$ of an LCA group $G$, we define its annihilator as

$$
H^{\perp}=\{\omega \in \widehat{G} \mid \omega(x)=1 \text { for all } x \in H\} .
$$

The annihilator is a closed subgroup in $\widehat{G}$, and if $H$ is a closed subgroup itself, then $\widehat{H} \cong \widehat{G} / H^{\perp}$ and $\widehat{G / H} \cong H^{\perp}$. These relations show that for a closed subgroup $H$ the quotient $G / H$ is compact if and only if $H^{\perp}$ is discrete.

For a closed subgroup $H$ of $G$ we Let $\pi_{H}: G \rightarrow$ $G / H, \pi_{H}(x)=x+H$ be the canonical map from $G$ onto $G / H$. If $f \in L^{1}(G)$, then the function $\dot{x} \mapsto \int_{H} f(x+h) d h$, $\dot{x}=\pi_{H}(x)$ defined almost everywhere on $G / H$, is integrable. Furthermore, when two of the Haar measures on $G, H$ and $G / H$ are given, then the third can be normalized such that Weil's formula

$$
\int_{G} f(x) d x=\int_{G / H} \int_{H} f(x+h) d \mu_{H}(h) d \mu_{G / H}(\dot{x})
$$

holds. If, in addition, $H$ is co-compact, i.e., the quotient group is compact, we define the size of $H$ as

$$
s(H):=\int_{G / H} 1 d \mu_{G / H}(\dot{x}) .
$$

We will assume the following setup for any given closed, co-compact subgroup $H$ of $G$. We fix a measure on $G$ and on
$G / H$. Since $G / H$ is compact, all Haar measures are of the form $c \cdot \tilde{\mu}_{G / H}$, where $c>0$ and $\tilde{\mu}_{G / H}$ denotes the normalized measure $\tilde{\mu}_{G / H}(G / H)=1$. With this convention we have $c=$ $d(H)$. By the property of dual measures, this means that the discrete subgroup $H^{\perp} \subset \widehat{G}$ is equipped with the counting measure multiplied by the constant $1 / d(H)$.

Example 2 (lattices). Let $H=P \mathbb{Z}^{n}, P \in \mathrm{GL}_{d}(\mathbb{R})$, be a subgroup of $G=\mathbb{R}^{n}$. Clearly, $H$ is both discrete and cocompact, hence a lattice. As measure on $\mathbb{R}^{n} / P \mathbb{Z}^{n}$ we pick the normalized measure multiplied with $|\operatorname{det} P|$. With this choice the size of $H$ becomes $d(H)=|\operatorname{det} P|$, and the measure on $H$ is the (normalized) counting measure. Note that $\mathbb{R}^{n} / P \mathbb{Z}^{n}$ is can be identified with the fundamental domain $F:=P\left([0,1)^{n}\right)$ and that $d(H)$ is its volume. Hence, Weil's formula reads

$$
\int_{\mathbb{R}^{n}} f(x) d x=|\operatorname{det} P| \int_{F} \sum_{h \in P \mathbb{Z}^{n}} f(x+h) d x
$$

Example 3 (co-compact subgroups). Let $H=\mathbb{R}^{s} \times P \mathbb{Z}^{d-s} \subset$ $G:=\mathbb{R}^{n}$, where $P \in \mathrm{GL}_{d-s}(\mathbb{R})$ and $s>0$. The subgroup $H$ is no longer discrete (but it is co-compact) so we cannot choose the counting measure on $H$. It is therefore natural to equip the compact subgroup $\mathbb{R}^{n} / H$ the normalized Haar measure. With this choice we see that $d(H)=1$. The annihilator of $H$ is discrete and is given by

$$
H^{\perp}=\{0\}^{s} \times\left(P^{T}\right)^{-1} \mathbb{Z}^{d-s} .
$$

The Haar measure on $H^{\perp}$ is simply the (normalized) counting measure.

Setting $F:=\{0\}^{s} \times P\left([0,1)^{d-s}\right)$, Weil's formula reads

$$
\int_{\mathbb{R}^{n}} f(x) d x=\int_{F} \sum_{h \in H} f(x+h) d x
$$

From Example 2 and 3 we see that the quantity $d(H)$ generalizes the usual concept of lattice size [12].

Definition III.1. Let $\Gamma \subset \widehat{G}$ and $\Lambda \subset G$ be closed, cocompact subgroups. A co-compact Gabor system in $L^{2}(G)$ with generator $g \in L^{2}(G)$ is a family of functions of the form

$$
\mathcal{G}(g, \Lambda, \Gamma):=\left\{E_{\gamma} T_{\lambda} g\right\}_{\gamma \in \Gamma, \lambda \in \Lambda} .
$$

We need the following characterizations of dual Gabor frames; it follows from a more general result on so-called characterizing equations for generalized translation invariant systems from [13].

Theorem III.2 ([13]). Let $\Gamma \subset \widehat{G}$ and $\Lambda \subset G$ be closed, cocompact subgroups. Suppose that the two systems $\mathcal{G}(g, \Lambda, \Gamma)$ and $\mathcal{G}(h, \Lambda, \Gamma)$ are Bessel systems. Then the following statements are equivalent:

(i) $\mathcal{G}(g, \Lambda, \Gamma)$ and $\mathcal{G}(h, \Lambda, \Gamma)$ are dual frames for $L^{2}(G)$,

(ii) for each $\alpha \in \Gamma^{\perp}$ we have

$$
s_{\alpha}(x):=\int_{\Lambda} \overline{g(x-\lambda-\alpha)} h(x-\lambda) d \mu_{\Lambda}(\lambda)=d(\Gamma) \delta_{\alpha, 0},
$$


for a.e. $x \in G$.

For $g, h \in L^{2}(G)$ assume that two co-compact Gabor systems $\mathcal{G}(g, \Lambda, \Gamma)$ and $\mathcal{G}(h, \Lambda, \Gamma)$ are Bessel systems with bounds $B_{g}$ and $B_{h}$, respectively. By an application of CauchySchwarz' inequality and [13, Proposition 3.3], we see that $s_{g, h, \alpha} \in L^{\infty}(G)$; to be precise:

$$
\left|s_{g, h, \alpha}(x)\right| \leq B_{g}^{1 / 2} B_{h}^{1 / 2} \quad \text { for a.e. } x \in G .
$$

Note that the function $s_{g, h, \alpha}: G \rightarrow \mathbb{C}$ is $\Lambda$-periodic. Since $G / \Lambda$ is compact and $s_{\alpha}$ uniformly bounded, we can consider $s_{g, h, \alpha}$ as a function in $L^{2}(G / \Lambda)$. Its Fourier series is given by

$$
s_{g, h, \alpha}(x)=\frac{1}{d(\Lambda)} \sum_{\beta \in \Lambda^{\perp}} c_{\alpha, \beta} \beta(x),
$$

where the Fourier coefficients $c_{\alpha, \beta}$ are given by:

$$
\begin{aligned}
c_{\alpha, \beta} & =\int_{G / \Lambda} s_{g, h, \alpha}(\dot{x}) \overline{\beta(\dot{x})} d \dot{x} \\
& =\int_{G / \Lambda} \int_{\Lambda} \overline{g(x-\lambda-\alpha)} h(x-\lambda) \overline{\beta(x-\lambda)} d \lambda d \dot{x} \\
& =\int_{G} h(x) \overline{\beta(x) g(x-\alpha)} d x=\left\langle h, E_{\beta} T_{\alpha} g\right\rangle .
\end{aligned}
$$

In the third equality we have used Weil's formula.

\section{Duality Results}

For a co-compact Gabor system $\mathcal{G}(g, \Lambda, \Gamma)$ its adjoint Gabor system is given by $\mathcal{G}\left(g, \Gamma^{\perp}, \Lambda^{\perp}\right)$. The time-frequency shifts in a Gabor system and its adjoint system are characterized by the fact that they commute [14, Section 3.5.3], [15, Lemma 7.4.1]. That is, for $(\lambda, \gamma) \in \Lambda \times \Gamma$ the point $(\alpha, \beta) \subset G \times \widehat{G}$ belongs to $\Gamma^{\perp} \times \Lambda^{\perp}$ if and only if

$$
\left(E_{\gamma} T_{\lambda}\right)\left(E_{\beta} T_{\alpha}\right)=\left(E_{\beta} T_{\alpha}\right)\left(E_{\gamma} T_{\lambda}\right) .
$$

The first result below shows Bessel duality between a Gabor system $\mathcal{G}(g, \Lambda, \Gamma)$ its adjoint Gabor system. To be more precise, a co-compact Gabor system is a Bessel family if, and only if, the adjoint Gabor system is a Bessel sequence. The result generalizes a result in [14]; a proof can be found in [10].

Proposition IV.1 ([10]). Let $B>0$ and $g \in L^{2}(G)$ be given. Let $\Gamma$ and $\Lambda$ be closed, co-compact subgroups. Then $\mathcal{G}(g, \Lambda, \Gamma)$ is a Bessel family with bound $B$ if, and only if, $\mathcal{G}\left(g, \Gamma^{\perp}, \Lambda^{\perp}\right)$ is a Bessel sequence with bound $d(\Gamma) d(\Lambda) B$.

\section{A. Wexler-Raz biorthogonality relations}

We are now able to state and prove Wexler-Raz biorthogonality relations [3], [6] for co-compact Gabor systems in $L^{2}(G)$.

Theorem IV.2 ([10]). Let $\Gamma$ and $\Lambda$ be closed, co-compact subgroups. Let $g, h \in L^{2}(G)$ and assume that two Gabor systems $\mathcal{G}(g, \Lambda, \Gamma)$ and $\mathcal{G}(h, \Lambda, \Gamma)$ are Bessel families. The two Gabor systems are dual frames if, and only if,

$$
\left\langle E_{\beta} T_{\alpha} h, E_{\beta^{\prime}} T_{\alpha^{\prime}} g\right\rangle=d(\Gamma) d(\Lambda) \delta_{\alpha, \alpha^{\prime}} \delta_{\beta, \beta^{\prime}}
$$

for all $\alpha, \alpha^{\prime} \in \Gamma^{\perp}$ and $\beta, \beta^{\prime} \in \Lambda^{\perp}$.

Proof. Assume that the two Gabor systems are dual frames. Then, for each $\alpha \in \Gamma^{\perp}$, we have $s_{g, h, \alpha}(x)=d(\Gamma) \delta_{\alpha, 0}$ for a.e. $x \in G$. Recall that the Fourier series of $s$ is:

$$
s_{g, h, \alpha}(x)=\frac{1}{d(\Lambda)} \sum_{\beta \in \Lambda^{\perp}}\left\langle h, E_{\beta} T_{\alpha} g\right\rangle \beta(x) .
$$

By uniqueness of the Fourier coefficients, it follows that

$$
\left\langle h, E_{\beta} T_{\alpha} g\right\rangle=d(\Gamma) d(\Lambda) \delta_{\beta, 1} \delta_{\alpha, 0}
$$

for all $\alpha \in \Gamma^{\perp}$ and $\beta \in \Lambda^{\perp}$. On the other hand, by unitarity of the translation and modulation operator, we have

$$
\left\langle h, E_{\beta} T_{\alpha} g\right\rangle=\left\langle E_{\beta^{\prime}} T_{\alpha^{\prime}} h, \overline{\beta(\alpha)} E_{\beta^{\prime} \beta} T_{\alpha^{\prime}+\alpha} g\right\rangle
$$

using (4). Combining the two last equations yields (7). The converse direction is now immediate.

\section{B. The duality principle}

The following result on Riesz sequences in abstract Hilbert spaces is a subspace variant of [16, Theorem 3.4.4] and [17, Theorem 7.13]. It is a scaled version of a result in [10] whose proof we follow.

Theorem IV.3. Let $A, B, c>0$, and let $\left\{\varphi_{k}\right\}$ be a sequence in a Hilbert space. Then the following statements are equivalent:

(a) $\left\{\varphi_{k}\right\}$ is a Riesz sequence with lower bound $c A$ and upper bound $c B$,

(b) $\left\{\varphi_{k}\right\}$ is a Bessel sequence with bound $c B$ and possesses a biorthogonal system $\left\{\psi_{k}\right\}$ normalized so that $\left\langle\varphi_{k}, \psi_{n}\right\rangle=$ $c \delta_{n, k}$, which is also a Bessel family with bound $c A^{-1}$.

Proof. Assume first $c=1$. Assume that (a) holds. Set $V=$ $\overline{\operatorname{span}}\left\{\varphi_{k}\right\}$. Let $\left\{\psi_{k}\right\}$ be the unique dual Riesz sequence of $\left\{\varphi_{k}\right\}$ in $V$ so that $\operatorname{span}\left\{\psi_{k}\right\}=V$. This implies (b).

Assume that (b) holds. Since $\left\{\varphi_{k}\right\}$ and $\left\{\psi_{k}\right\}$ are biorthogonal, it follows that

$$
\varphi_{j}=\sum_{k=1}^{\infty}\left\langle\varphi_{j}, \psi_{k}\right\rangle \varphi_{k}
$$

for all $j$. By linearity, we have, for any $f \in \operatorname{span}\left\{\varphi_{k}\right\}$,

$$
f=\sum_{k=1}^{\infty}\left\langle f, \psi_{k}\right\rangle \varphi_{k} .
$$

This formula extends to $\overline{\operatorname{span}}\left\{\varphi_{k}\right\}$ by continuity. Now, for any $f \in \overline{\operatorname{span}}\left\{\varphi_{k}\right\}$, we have

$$
\begin{aligned}
\|f\|^{2} & =|\langle f, f\rangle|=\left|\sum_{k=1}^{\infty}\left\langle f, \psi_{k}\right\rangle\left\langle\varphi_{k}, f\right\rangle\right| \\
& \leq\left(\sum_{k=1}^{\infty}\left|\left\langle f, \psi_{k}\right\rangle\right|^{2} \sum_{k=1}^{\infty}\left|\left\langle f, \varphi_{k}\right\rangle\right|^{2}\right)^{1 / 2} \\
& \leq A^{-1 / 2}\|f\|\left(\sum_{k=1}^{\infty}\left|\left\langle f, \varphi_{k}\right\rangle\right|^{2}\right)^{1 / 2} .
\end{aligned}
$$

We see that $\left\{\varphi_{k}\right\}$ is a frame sequence with lower frame bound $A$; by assumption the upper frame bound is $B$. By the fact that 
$\left\{\varphi_{k}\right\}$ possesses a biorthogonal sequence, it follows that $\left\{\varphi_{k}\right\}$ is, in fact, a Riesz sequence with the same bounds.

For $c \neq 1$ repeat the above computations for $\left\{\frac{1}{c^{1 / 2}} \varphi_{k}\right\}$ and $\left\{\frac{1}{c^{1 / 2}} \psi_{k}\right\}$

We now state and prove the duality principle for co-compact Gabor systems.

Theorem IV.4 ([10]). Let $g \in L^{2}(G)$. Let $\Gamma$ and $\Lambda$ be closed, co-compact subgroups. Then $\mathcal{G}(g, \Lambda, \Gamma)$ is a frame for $L^{2}(G)$ with bounds $A$ and $B$ if, and only if, $\mathcal{G}\left(g, \Gamma^{\perp}, \Lambda^{\perp}\right)$ is a Riesz sequence with bounds $d(\Gamma) d(\Lambda) A$ and $d(\Gamma) d(\Lambda) B$.

Proof. Let $\mathcal{G}(g, \Lambda, \Gamma)$ be a frame with bounds $A$ and $B$. The canonical dual frame $\mathcal{G}\left(S^{-1} g, \Lambda, \Gamma\right)$ has bounds $B^{-1}$ and $A^{-1}$. By Proposition IV.1, the sequences $\mathcal{G}\left(g, \Gamma^{\perp}, \Lambda^{\perp}\right)$ and $\mathcal{G}\left(S^{-1} g, \Gamma^{\perp}, \Lambda^{\perp}\right)$ are Bessel families with bound $d(\Gamma) d(\Lambda) B$ and $d(\Gamma) d(\Lambda) A^{-1}$, respectively. By Wexler-Raz biorthogonal relations, these two families are biorthogonal:

$$
\left\langle E_{\beta} T_{\alpha} g, E_{\beta^{\prime}} T_{\alpha^{\prime}} S^{-1} g\right\rangle=d(\Gamma) d(\Lambda) \delta_{\alpha, \alpha^{\prime}} \delta_{\beta, \beta^{\prime}}
$$

Hence, by Theorem IV.3 with $c=d(\Gamma) d(\Lambda)$, we conclude that $\mathcal{G}\left(g, \Gamma^{\perp}, \Lambda^{\perp}\right)$ is a Riesz sequence with bounds $d(\Gamma) d(\Lambda) A$ and $d(\Gamma) d(\Lambda) B$.

Conversely, suppose $\mathcal{G}\left(g, \Gamma^{\perp}, \Lambda^{\perp}\right)$ is a Riesz sequence with bounds $d(\Gamma) d(\Lambda) A$ and $d(\Gamma) d(\Lambda) B$. The dual Riesz sequence of $\mathcal{G}\left(g, \Gamma^{\perp}, \Lambda^{\perp}\right)$ is of the form $\mathcal{G}\left(h, \Gamma^{\perp}, \Lambda^{\perp}\right)$ for some $h \in$ $L^{2}(G)$ and has bounds $(d(\Gamma) d(\Lambda) B)^{-1}$ and $(d(\Gamma) d(\Lambda) A)^{-1}$. Using Proposition IV.1 we see that $\mathcal{G}(g, \Lambda, \Gamma)$ has Bessel bound $B$. On the other hand, $\mathcal{G}(h, \Lambda, \Gamma)$ has Bessel bound $A^{-1}(d(\Gamma) d(\Lambda))^{-2}$. By Wexler-Raz biorthogonal relations, $\mathcal{G}(g, \Lambda, \Gamma)$ and $\mathcal{G}(d(\Gamma) d(\Lambda) h, \Lambda, \Gamma)$ are dual frames. Since the upper frame bound of $\mathcal{G}(h, \Lambda, \Gamma)$ is $A^{-1}(d(\Gamma) d(\Lambda))^{-2}$, it is immediate the upper frame bound of $\mathcal{G}\left((d(\Gamma) d(\Lambda))^{-1} h, \Lambda, \Gamma\right)$ is $A^{-1}$. Hence, by a computation as in (8), we see that $A$ is a lower frame bound for $\mathcal{G}(g, \Lambda, \Gamma)$.

Since a Riesz sequence with equal upper and lower bounds is an orthogonal sequence, we have the following corollary of Theorem IV.4.

Corollary IV.5. Let $\Gamma$ and $\Lambda$ be closed, co-compact subgroups. A Gabor system $\mathcal{G}(g, \Lambda, \Gamma)$ is a tight frame if, and only if, $\mathcal{G}\left(g, \Gamma^{\perp}, \Lambda^{\perp}\right)$ is an orthogonal system. In these cases, the frame bound is given by $A=\|g\|^{2}(d(\Gamma) d(\Lambda))^{-1}$.

Example 4. In $L^{2}\left(\mathbb{R}^{d}\right)$ we consider the co-compact Gabor system

$\mathcal{G}(g, \Lambda, \Gamma) \quad$ with $\Lambda=A\left(\mathbb{R}^{s} \times \mathbb{Z}^{d-s}\right), \quad \Gamma=B\left(\mathbb{R}^{r} \times \mathbb{Z}^{d-r}\right)$

for some choice of $0 \leq r, s \leq d$. The duality principle says that $\mathcal{G}(g, \Lambda, \Gamma)$ is a continuous frame with bounds $A$ and $B$, if and only if the adjoint system

$$
\mathcal{G}\left(g,\left(B^{T}\right)^{-1}\left(\{0\}^{r} \times \mathbb{Z}^{d-r}\right),\left(A^{T}\right)^{-1}\left(\{0\}^{s} \times \mathbb{Z}^{d-s}\right)\right)
$$

is a Riesz sequence with bounds $A$ and $B$. If $s=r=d$, the adjoint system is simply $\{g\}$ which is a Riesz sequence with bound $\|g\|^{2}$. Hence, in this case, the Gabor system $\mathcal{G}(g, \Lambda, \Gamma)$ associated with the short-time Fourier transform is a tight frame for any $g \neq 0$.

Remark 1. By choosing the normalized measure on $G / \Lambda$ and $\widehat{G} / \Gamma$, we have that $d(\Lambda)=d(\Gamma)=1$. Hence, in this case, the Wexler-Raz relations become a biorthonormality condition, while in the duality principle we have preservation of bounds between the frame and the Riesz sequence. This shows that choosing normalized measures on $G / \Lambda$ and $\widehat{G} / \Gamma$ in some sense is very natural. On the other hand, this choice does not agree with the standard choice of measures in lattice Gabor analysis.

\section{REFERENCES}

[1] I. Daubechies, H. J. Landau, and Z. Landau, "Gabor time-frequency lattices and the Wexler-Raz identity," J. Fourier Anal. Appl., vol. 1, no. 4, pp. 437-478, 1995.

[2] A. J. E. M. Janssen, "Signal analytic proofs of two basic results on lattice expansions," Appl. Comput. Harmon. Anal., vol. 1, no. 4, pp. 350-354, 1994.

[3] - "Duality and biorthogonality for Weyl-Heisenberg frames," $J$. Fourier Anal. Appl., vol. 1, no. 4, pp. 403-436, 1995.

[4] R. Tolimieri and R. S. Orr, "Characterization of Weyl-Heisenberg frames via poisson summation relationships," Proc. Internat. Conf. Acoust. Speech Signal Process., vol. 4, pp. 277-280, 1992.

[5] — "Poisson summation, the ambiguity function, and the theory of Weyl-Heisenberg frames," J. Fourier Anal. Appl., vol. 1, no. 3, pp. 233-247, 1995.

[6] J. Wexler and S. Raz, "Discrete Gabor expansions," Signal Processing, vol. 21 , no. 3, pp. 207-220, Nov 1990.

[7] A. J. E. M. Janssen, "The duality condition for Weyl-Heisenberg frames," in Gabor analysis and algorithms, ser. Appl. Numer. Harmon. Anal. Boston, MA: Birkhäuser Boston, 1998, pp. 33-84.

[8] A. Ron and Z. Shen, "Weyl-Heisenberg frames and Riesz bases in $L_{2}\left(\mathbf{R}^{d}\right)$," Duke Math. J., vol. 89, no. 2, pp. 237-282, 1997.

[9] - "Frames and stable bases for shift-invariant subspaces of $L_{2}\left(\mathbf{R}^{d}\right)$," Canad. J. Math., vol. 47, no. 5, pp. 1051-1094, 1995.

[10] M. S. Jakobsen and J. Lemvig, "Co-compact gabor systems on locally compact abelian groups,", J. Fourier Anal. Appl., to appear, DOI: 10.1007/s00041-015-9407-0.

[11] E. Hewitt and K. A. Ross, Abstract harmonic analysis. Vol. II: Structure and analysis for compact groups. Analysis on locally compact Abelian groups, ser. Die Grundlehren der mathematischen Wissenschaften, Band 152. New York: Springer-Verlag, 1970.

[12] J. W. S. Cassels, An introduction to the geometry of numbers, ser. Classics in Mathematics. Berlin: Springer-Verlag, 1997, corrected reprint of the 1971 edition.

[13] M. S. Jakobsen and J. Lemvig, "Reproducing formulas for generalized translation invariant systems on locally compact abelian groups," Trans. Amer. Math. Soc., to appear.

[14] H. G. Feichtinger and G. Zimmermann, "A Banach space of test functions for Gabor analysis," in Gabor analysis and algorithms, ser. Appl. Numer. Harmon. Anal. Boston, MA: Birkhäuser Boston, 1998, pp. 123-170.

[15] K. Gröchenig, Foundations of Time-Frequency Analysis, ser. Appl. Numer. Harmon. Anal. Birkhäuser, 2001.

[16] O. Christensen, Frames and bases, ser. Applied and Numerical Harmonic Analysis. Boston, MA: Birkhäuser Boston Inc., 2008, an introductory course.

[17] C. Heil, A basis theory primer, expanded ed., ser. Applied and Numerical Harmonic Analysis. Birkhäuser/Springer, New York, 2011. 